\title{
RELAÇÃO ENTRE DESMATAMENTO E PRECIPITAÇÃO NOS MUNICÍPIOS DE NOVA UBIRATÃ - MT E SÃO FELIX DO ARAGUAIA - MT NA BACIA DO ALTO XINGU
}

\author{
Leonardo Auge Levyman $^{(a)}$, Tatiane da Silva Gregório ${ }^{(b)}$, Daniela Fernanda Silva Fuzzo ${ }^{(c)}$, \\ Edson Luís Piroli ${ }^{(\mathrm{d})}$
}

(a) Graduando em Geografia, Universidade Estadual Paulista - UNESP - Campus de Ourinhos - E-mail: leoauge@ hotmail.com; taty_gregorio@hotmail.com.

(b) Graduando em Geografia, Universidade Estadual Paulista - UNESP - Campus de Ourinhos - E-mail: taty_gregorio@hotmail.com

(c) Professor Assistente Doutor do curso de graduação em Geografia da Unesp - Campus Experimental de Ourinhos e do Programa de Pós-Graduação em Geografia da Faculdade de Ciências e Tecnologia da Unesp Campus de Presidente Prudente. E-mail: elp@ ourinhos.unesp.br

(d) Profa. Dra. Subs, Universidade Estadual Paulista - UNESP - Campus de Ourinhos. Email: daniela@ourinhos.unesp.br

\section{Eixo: GEOTECNOLOGIAS E MODELAGEM ESPACIAL EM GEOGRAFIA FÍSICA}

\begin{abstract}
RESUMO
Na Geografia física, a geotecnologia vem ganhando cada vez mais espaço, se tornando uma ferramenta indispensável para monitoramentoe estudos de grandes porções territoriais. O presente estudo visa entender se a questão do desmatamento provocado por ações antrópicas em larga escala principalmente na região centro oeste e norte de nosso país, onde a área de estudo está inserida (Bacia do Alto Xingu), afeta em algum sentido a precipitação da região, alterando o ciclo hidrológico que por sua vez gera um possível desequilíbrio no meio ambiente. O objetivo deste trabalho foi analisar o que o avanço do desmatamento tem influenciado no ritmo pluviométrico dos municípios de Nova Ubiratã eSão Felix do Araguaia, no período de 2000 a 2015.
\end{abstract}

Palavras chave: TRMM, Alto Xingu, Desmatamento, Precipitação, Mato Grosso.

\section{Introdução}

O avanço do agronegócio na região é claramente, uma consequência de um contexto mundial, no qual mercados externos pressionam e incentivam a produção de determinados produtos (ARAUJO \& PONTE, 2015). Novas tecnologias permitiram que a região fosse ocupada por cultivos de grãos e pastagens, acarretando o início da chamada expansão da fronteira agrícola, que apesar dos impactos positivos na economia do país, também foi responsável pelo desmatamento de grandes áreas da Floresta Amazônica.

Com isso, o desmatamento deixa rastros irrecuperáveis em seu caminho, não só em danos físicos visíveis, mas também existe o impacto social que ocorre na mesma proporção. Uma das regiões que vem apresentando grandes modificações devido a essa expansão é a Bacia do Alto Xingu, que apresentou forte aumento nos índices de desmatamento, onde a taxa de desmatamento está próxima dos $100 \mathrm{~km}^{2}$ (Instituto Socioambiental - ISA, 2016). 
Porém esse valor vem diminuindo nos últimos anos, a queda na taxa de desmatamento está relacionada principalmente a segunda fase do PPCDAm (Plano de Prevenção e Controle do Desmatamento na Amazônia Legal), que focou em ações de monitoramento (Sistema DETER - Sistema de detecção de desmatamento em tempo real) e comando e controle, onde a operação Arco de Fogo da Policia Federal é o exemplo como um dos exemplos com mais evidencia.

Sob essa premissa Netto (2002), corrobora destacando que dentre os problemas ambientais existentes, decorrente do desmatamento existe forte relação entre a intensidade das chuvas nessas regiões, segundo o autor nos últimos anos tem ocorrido alterações significativas embora localizadas, nos fluxos de água, energia, carbono, e ciclagem de nutrientes e na composição da atmosfera foram causadas por desmatamentos e queimadas.

Desta forma, se faz necessário a utilização de uma alternativa viável, rápida e de baixo custo para o monitoramento dessas áreas, por meio da utilização de dados orbitais, como por exemplo, dados obtidos pelo satélite TRMM (Tropical RainfallMeasuringMission - NASA), utilizados para monitorar em maior escala a ocorrência de chuva na superfície terrestre, principalmente em regiões com escassez de dados meteorológicos de superfície. Segundo Silva-Fuzzo et al., (2015) o satélite TRMM é uma parceria entre a NASA (NationalAeronauticsand Space Administration) e a JAXA (Agência Japonesa de Exploração Aeroespacial), e seu objetivo específico é monitorar e estudar a precipitação em áreas tropicais.

O objetivo deste trabalho foi analisar quanto o avanço do desmatamento tem influenciado no ritmo pluviométrico, nos municípios de Nova Ubiratã e São Felix do Araguaia, no período de 2000 a 2015, localizados na bacia do Alto Xingu, no estado do Mato Grosso.

\section{$2 \quad$ Material e métodos}

A Bacia do Alto Xingu está localizada no estado do Mato Grosso, entre a latitude $11^{\circ} 32^{\prime}$ S e longitude $53^{\circ} 20^{\prime} \mathrm{W}$, conhecida como a região das cabeceiras do rio Xingu, com uma extensão de 17,7 milhões de hectares (ISA, 2011), o que equivale a $34 \%$ de toda a Bacia do Xingu, os municípios analisados foram

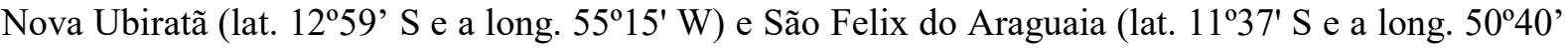
W) situados nos limites da bacia. (Figura 1). 

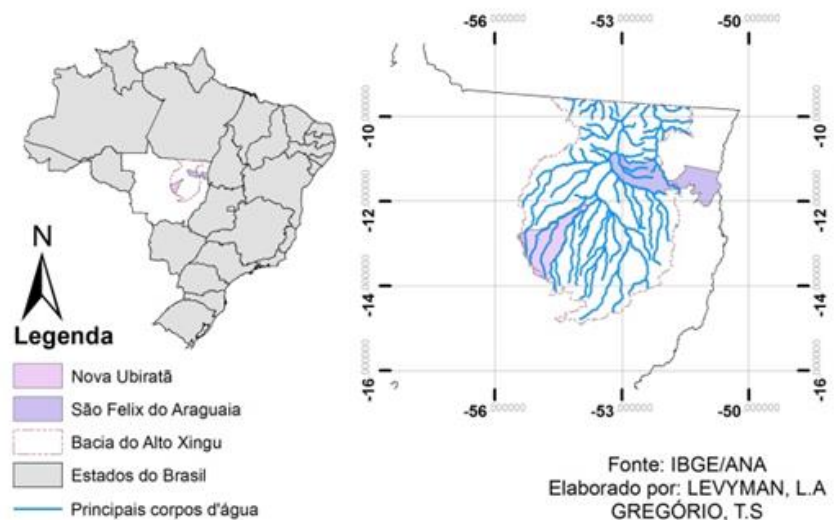

Figura 1 - Localização da área de estudo

Os dados referentes à precipitação foram obtidos por meio do satélite meteorológico TRMM, pelo sensor PR (Precipitation Radar), que foi o primeiro instrumento desenhado para gerar mapas tridimensionais das estruturas das chuvas, com o produto 3B42-V7, essa interface é projetada para visualização e análise de dados mensais acumulados. Os dados vêm em formato "nc" (formato que carrega informações multidimensionais de dados climáticos para plataformas) e convertidos para formato GeoTiff pelo Software Arcgis10.5, com resolução espacial de $0,25^{\circ}$ x $0,25^{\circ}$. Esses dados estão disponibilizados gratuitamente online através do website da NASA: < http://trmm.gsfc.nasa.gov/data_dir/data.html>.

Os dados vetoriais referentes ao desmatamento foram obtidos pelo site do I3Geo do Ministério do Meio Ambiente, <http://mapas.mma.gov.br/i3geo/datadownload.htm>, e os dados tabulares sobre desmatamento foram obtidos no site do Sistema PRODES do INPE, link: <http://www.dpi.inpe.br/prodesdigital/prodesmunicipal.php>. Desta forma, primeiramente foi feito o levantamento dos dados de desmatamento, para os dois municípios, e consequentemente sua relação com a precipitação, para os anos de 2000, 2005, 2010 e 2015, para os dois municípios. Com intuito de analisar a precipitação na região de estudo, foi selecionado o mês de dezembro, que conforme Souza et al., (2013) as regiões do médio-norte e norte do estado do Mato Grosso, caracterizam-se no inverno (junho, julho e agosto) quando ocorrem as maiores amplitudes térmicas diárias e durante os meses de verão ou período chuvoso (dezembro, janeiro, fevereiro e março).

\section{Discussão e Análise}

Os dados apresentados na Tabela 1 indicam os valores de desmatamento em $\mathrm{km}^{2}$, no período de 2000 a 2015, nos dois municípios analisados. O município de São Felix do Araguaia tem o ano de 2008 com o maior valor de desmatamento chegando à $210,1 \mathrm{~km}^{2}$, e o menor o ano $2010 \mathrm{com} 6,3 \mathrm{~km}^{2}$, e em Nova Ubiratã, o maior índice foi para o ano de 2004 e o menor 2013, com 382,9 km² e 9,7 km², 
XVII Simpósio Brasileiro de Geografia Fisica Aplicada

I Congresso Nacional de Geografia Física

\section{OS DESAFIOS DA GEOGRAFIA FÍSICA NA FRONTEIRA DO CONHECIMENTO \\ Instituto de Geociências - Unicamp \\ Campinas - SP \\ 28 de Junho à 02 de Julho de 2017}

respectivamente. Também vale destacar que nos dois municípios os valores apresentam uma considerada redução desses valores a partir do ano de 2009.

Tabela 1 - Taxa de desmatamento por município (2000/2015). Fonte: DETER do INPE/ Ministério do Meio Ambiente.

Taxa de desmatamento do município de São Felix do Araguaia -MT (2000 - 2015)

\begin{tabular}{|c|c|c|c|c|c|}
\hline Total desmatado até 2000 & 2001 & 2002 & 2003 & 2004 & 2005 \\
\hline$+3396.5 \mathrm{Km}^{2}$ & $+103.8 \mathrm{Km}^{2}$ & $+72.4 \mathrm{Km}^{2}$ & $+159.1 \mathrm{Km}^{2}$ & $+189.8 \mathrm{Km}^{2}$ & $+163.1 \mathrm{Km}^{2}$ \\
\hline 2006 & 2007 & 2008 & 2009 & 2010 & 2011 \\
\hline$+50.9 \mathrm{Km}^{2}$ & $+41.4 \mathrm{Km}^{2}$ & $+210.1 \mathrm{Km}^{2}$ & $+17.6 \mathrm{Km}^{2}$ & $+6.3 \mathrm{Km}^{2}$ & $+19.1 \mathrm{Km}^{2}$ \\
\hline 2012 & 2013 & 2014 & \multicolumn{3}{|c|}{2015} \\
\hline$+17.4 \mathrm{Km}^{2}$ & $+16.4 \mathrm{Km}^{2}$ & $+22.8 \mathrm{Km}^{2}$ & \multicolumn{3}{c|}{$+20.5 \mathrm{Km}^{2}$} \\
\hline
\end{tabular}

Taxa de desmatamento do município de Nova Ubiratã -MT (2000 - 2015)

\begin{tabular}{|c|c|c|c|c|c|}
\hline Total desmatado até 2000 & 2001 & 2002 & 2003 & 2004 & 2005 \\
\hline $2446.4 \mathrm{Km}^{2}$ & $+159.4 \mathrm{Km}^{2}$ & $+323.5 \mathrm{Km}^{2}$ & $+367.7 \mathrm{Km}^{2}$ & $+382.9 \mathrm{Krriz}$ & $+254.3 \mathrm{Km}^{2}$ \\
\hline 2006 & 2007 & 2008 & 2009 & 2010 & 2011 \\
\hline$+72.7 \mathrm{Km}^{2}$ & $+19.8 \mathrm{Km}^{2}$ & $+141.1 \mathrm{Km}^{2}$ & $+30.5 \mathrm{Km}^{2}$ & $+22.8 \mathrm{Km}^{2}$ & $+94.3 \mathrm{Km}^{2}$ \\
\hline 2012 & 2013 & 2014 & \multicolumn{3}{|c|}{2015} \\
\hline$+19.8 \mathrm{Km}^{2}$ & $+9.7 \mathrm{Km}^{2}$ & $+15.6 \mathrm{Km}^{2}$ & \multicolumn{3}{|c|}{$+28.1 \mathrm{Km}^{2}$} \\
\hline
\end{tabular}

A partir disso, foram gerados primeiramente os mapas de precipitação para o mês de dezembro (Figura 2), com intuito de acompanhar o quantificar a precipitação nesse mês, foram analisados os anos de 2000, 2005, 2010, e 2015, nesse foi possível observar que os menores valores encontrados foram os anos de 2000 e 2015 com valores acumulados de 483 e $292 \mathrm{~mm}$ respectivamente, e os anos de 2005 e 2010 com os maiores valores, sendo que o município de são Felix do Araguaia apresentou maior concentração de pixels com maiores valores de chuva em relação a Nova Ubiratã.

Segundo Leivas et al., (2011) e Silva-Fuzzo et al., (2016) foram analisados a confiabilidade dos dados TRMMem relação aos dados observados em superfície e obtiveram bons resultados nessa estimativa, confirmando que podem ser utilizados como uma fonte alternativa de informações sobre a escassez de dados de estações de superfície.

Relacionando os dados de precipitação (mês/dez) do satélite TRMM, aos dados de desmatamento nos municípios (Tabela 2), foram observados que entre os anos de 2005 a 2010, houve uma grande diminuição do desmatamento devido às medidas tomadas pelos órgãos governamentais.

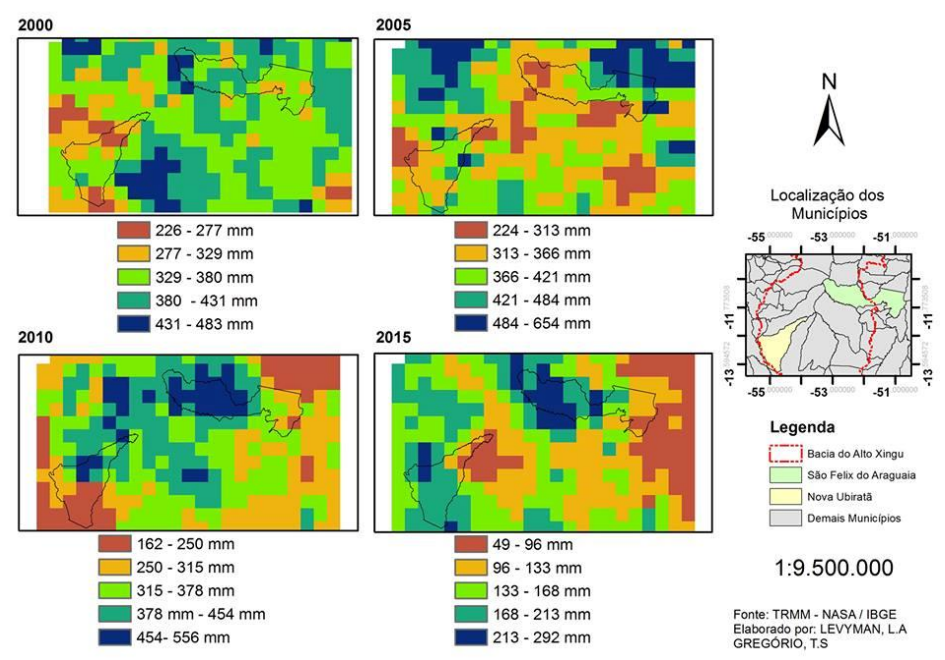


XVII Simpósio Brasileiro de Geografia Fisica Aplicada

I Congresso Nacional de Geografia Física

\section{OS DESAFIOS DA GEOGRAFIA FÍSICA NA FRONTEIRA DO CONHECIMENTO \\ Instituto de Geociências - Unicamp \\ Campinas - SP \\ 28 de Junho à 02 de Julho de 2017}

Figura 2 - Localização da área de estudo

Tabela 2 - Relação dos valores de precipitação (mm), com os valores de desmatamento $\left(\mathrm{km}^{2}\right)$. Fonte:

TRMM e DETER/INPE

\begin{tabular}{|c|c|c|c|c|c|}
\hline \multicolumn{3}{|c|}{ Nova Ubiratã - MT } & \multicolumn{3}{c|}{ São Felix do Araguaia - MT } \\
\hline Ano & $\begin{array}{c}\text { Média das chuvas } \\
\mathbf{m m} / \mathbf{m e ̂ s}\end{array}$ & $\begin{array}{c}\text { Desmatamento por ano } \\
\mathbf{k m}^{2}\end{array}$ & Ano & $\begin{array}{c}\text { Média das } \\
\text { chuvas mm/mês }\end{array}$ & $\begin{array}{c}\text { Desmatamento por ano } \\
\mathbf{k m}^{2}\end{array}$ \\
\hline $\mathbf{2 0 0 0}$ & 366 & $\begin{array}{c}\text { total desmatado anos } \\
\text { anteriores: } 2446 \mathrm{~km}^{2}\end{array}$ & $\mathbf{2 0 0 0}$ & 366 & $\begin{array}{c}\text { total desmatado anos } \\
\text { anteriores: } 3396 \mathrm{~km}^{2}\end{array}$ \\
\hline $\mathbf{2 0 0 5}$ & 390 & $254.3 \mathrm{~km}^{2}$ & $\mathbf{2 0 0 5}$ & 390 & $163 \mathrm{~km}^{2}$ \\
\hline $\mathbf{2 0 1 0}$ & 342 & $22.8 \mathrm{~km}^{2}$ & $\mathbf{2 0 1 0}$ & 342 & $6.3 \mathrm{~km}^{2}$ \\
\hline $\mathbf{2 0 1 5}$ & 144 & $28 \mathrm{~km}^{2}$ & $\mathbf{2 0 1 5}$ & 144 & $20.5 \mathrm{~km}^{2}$ \\
\hline
\end{tabular}

Obs: mês de chuva referente a dezembro

Fonte: TRMM - NASA / DETER INPE

Elaborado por: LEVYMAN, L.A, GREGORIO, T.S

Em seguida foram utilizadas as bases de dados vetoriais do MMA (Ministério do Meio Ambiente), com intuito de demarcar as áreas de desmatamento dentro dos municípios analisados, considerando o intervalo de tempo correspondente aos anos de 2004 a 2009, desta forma também foram comparados com os dados de precipitação dos anos que ocorreram maior diferença de nosso estudo, os anos de 2005 e 2010 (Figura 3 e 4).
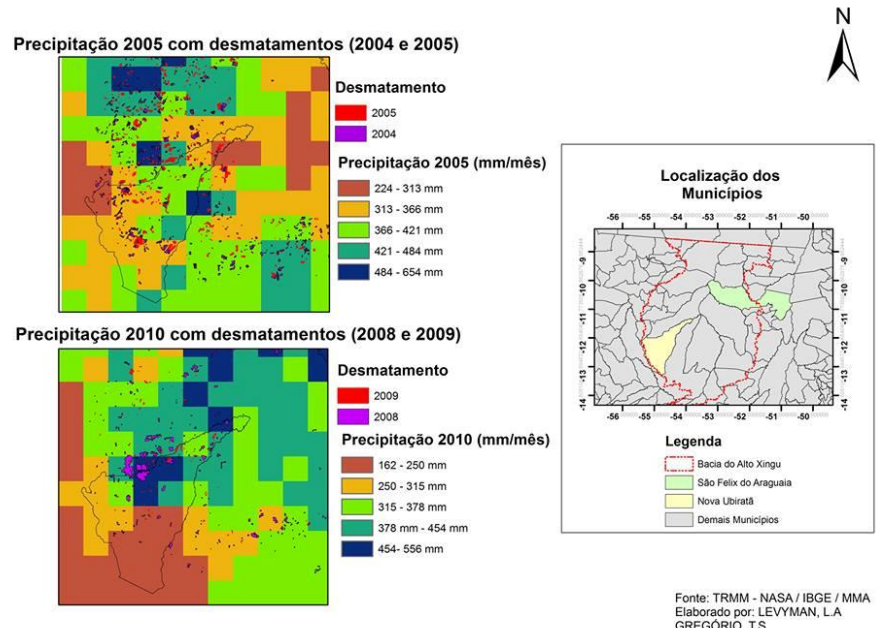

Figura 3 - Análise do desmatamento, em relação à precipitação no município de Nova Ubiratã-MT
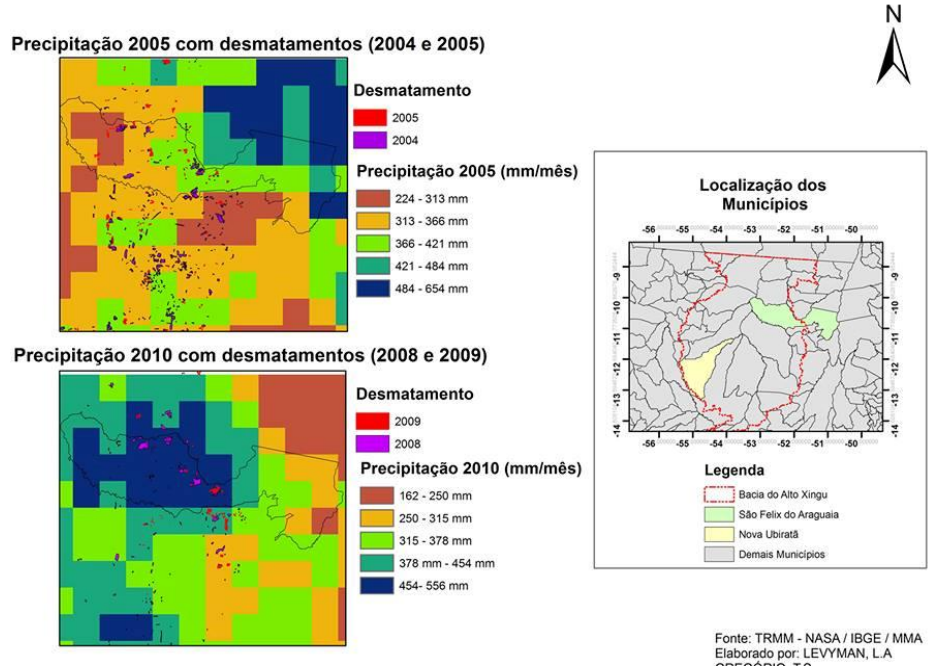

Figura 4. Análise do desmatamento, em relação à precipitação no município de São Felix do Xingu. 
As Figura 3 e 4 juntamente com a Tabela 2 evidenciam a relação das chuvas com o desmatamento principalmente nos anos de 2004-2005, onde a precipitação ocorreu com menor intensidade nas áreas na qual ocorriam os maiores valores de desmatamento.

\section{Conclusões}

Podemos concluir a ligação entre a precipitação e ao desmatamento. Os dados de precipitação estimados a partir de satélites como o TRMM podem ser uma alternativa eficiente e barata quando comparados a instrumentos de superfície. Porém futuramente serão necessários testes estatísticos mais aprofundados sob essa relação. $\mathrm{O}$ entendimento destes processos pode permitir um melhor planejamento de políticas públicas a fim de monitorar e mapear áreas mais suscetíveis a problemas ambientais.

\section{Bibliografia}

ARAUJO, R.C.; PONTE, M.X. Agronegócio na Amazônia: ameaças e oportunidades para o desenvolvimento sustentável na região. Revista de Agroambientais. Alta Floresta. MT. v.13, n.2, p. 101-114, 2015.

NETTO, P. E. A. Mudanças de uso de solo na Amazônia: implicações climáticas e na ciclagem de carbono. Manaus: Instituto Nacional de Pesquisas da Amazônia. INPA, 2002. (Reunião Científica)

SILVA-FUZZO et al. Modelagem agrometeorológica para estimativa de produtividade de soja para o vale do Médio Paranapanema-SP. Irriga Botucatu v. 20, n. 3, p. 490-501, julho - setembro, 2015. Botucatu - SP

SILVA-FUZZO, D.F. ROCHA.J.V. Validação dos dados de precipitação estimados pelo trmm, para o estado do paraná, e sua contribuição ao monitoramento agrometeorológico.Revista Formação (ONLINE) Vol. 3; n. 23, mai-ago/2016. p. 301- 316. 2016.

LEIVAS, J.F et al. Avaliação dos prognósticos de precipitação simulada pelo modelo BRAMS na Amazônia Ocidental na estação chuvosa. Acta Amaz., Manaus, v. 41, n. 3, 2011.

SOUZA, A.P.; MOTA, L.L.; ZAMDEI,T.; MARTIN, C.C.;ALMEIDA, F.T.; PAULINO, J. Classificação climática e balanço hídrico climatológico no estado de mato grosso, Instituto de Ciências Agrárias e Ambientais, Nativa, Sinop, v. 01, n. 01, p.34-43, out./dez., 2013.

ISA Instituto Socioambiental. Fique por dentro: a Bacia do Rio Xingu em Mato Grosso. 2010. -- (Série Cartô Brasil Socioambiental vol. 2) 